\title{
Magnetic impurities on the surface of a topological insulator
}

\author{
Qin $\mathrm{Liu}^{1}$, Chao-Xing $\mathrm{Liu}^{2}{ }^{3}$, Cenke $\mathrm{Xu}^{4}$, Xiao-Liang $\mathrm{Qi}^{3}$ and Shou-Cheng Zhang ${ }^{3}$ \\ ${ }^{1}$ Department of Physics, Fudan University, Shanghai 200433, China \\ ${ }^{2}$ Center for Advanced Study, Tsinghua University,Beijing, 100084, China \\ ${ }^{3}$ Department of Physics, McCullough Building, Stanford University, Stanford, CA 94305-4045 and \\ ${ }^{4}$ Department of Physics, Harvard University, Cambridge, Massachusetts 02138, USA
}

(Dated: August 18, 2008)

\begin{abstract}
The surface states of a topological insulator are described by an emergent relativistic massless Dirac equation in $2+1$ dimensions. In contrast to graphene, there is an odd number of Dirac points, and the electron spin is directly coupled to the momentum. We show that a magnetic impurity opens up a local gap and suppresses the local density of states. Furthermore, the Dirac electronic states mediate an RKKY interaction among the magnetic impurities which is always ferromagnetic, whenever the chemical potential lies near the Dirac point. These effects can be directly measured in STM experiments. We also study the case of quenched disorder through a renormalization group analysis.
\end{abstract}

PACS numbers: 75.30.Hx, 73.20.At, 73.20.-r, 72.25.Dc, 85.75.-d

Following the recent theoretical prediction and the experimental observation of the quantum spin Hall insulator state in two dimensions [1, 2, 3, , 4], the concept of a topological insulator (TI) in three dimensions has attracted a lot of interest $[\mathbf{5},[6,6,6]$. The electronic excitation spectrum of a time reversal invariant TI is fully gapped in the bulk, but there are gapless surface states described by the $2+1$ dimensional relativistic Dirac equation with an odd number of Dirac points. This property makes the surface system an extremely unusual $(2+1)$ d system, just like the $1+1$ dimensional "helical" edge states of quantum spin Hall insulators $[9,10]$. In fact, one can prove a general no-go theorem, which states that a two dimensional time reversal invariant lattice model can not have an odd number of Dirac points $[$ ] . For example, the familiar graphene model on a honeycomb lattice has four Dirac points 11, 12]. The surface states of a TI evade this no-go theorem since they describe the boundary of a three-dimensional lattice model, and a pair of Dirac points can be separated onto the two opposite surfaces. The Dirac points of a TI are thus stable and robust. They can not be destroyed by any time reversal invariant perturbations. In contrast, since the Dirac points of a two-dimensional lattice model occur in pairs, they can be pairwise annihilated by small perturbations. For example, a sublattice distortion in graphene can remove the Dirac points entirely.

Therefore, the surface states of a TI offer a unique platform to investigate the physics of robust Dirac points. In Ref [8, 13], it was pointed out that a time reversal breaking perturbation on the surface is the most natural way to reveal the topological properties of Dirac points. For this reason, we investigate the effects of magnetic impurities on the surface states of a TI. We consider the simplest case of a single Dirac point, described by the low-energy effective Hamiltonian

$\hat{H}_{0}=\sum_{k, \alpha, \beta} \psi_{k \alpha}^{\dagger} h_{\alpha \beta}(\mathbf{k}) \psi_{k \beta}, h_{\alpha \beta}(k)=\hbar v_{f}\left(k_{x} \sigma_{\alpha \beta}^{x}+k_{y} \sigma_{\alpha \beta}^{y}\right)$, where the $z$-direction is perpendicular to the surface and $v_{f}$ is the Fermi velocity. At first sight, this is exactly the 2D Dirac Hamiltonian at one nodal point of graphene which has been used successfully to describe its lowenergy physics 11, 12]. However, there is one important difference between these two cases. For graphene, the two components of the Dirac Hamiltonian describe the two sublattices or pseudo-spin degrees of freedom, while in the case of a TI, the two components describe the real electron spin, and are related to each other by time reversal. Therefore, we expect the coupling between magnetic impurities and electron spin to take the form

$\hat{H}_{e x}=\hat{H}_{e x}^{z}+\hat{H}_{e x}^{\|}=\sum_{\mathbf{r}} J_{z} s_{z}(\mathbf{r}) S_{z}(\mathbf{r})+J_{\|}\left(s_{x} S_{x}+s_{y} S_{y}\right)(\mathbf{r})$,

where $S_{i}(\mathbf{r})$ is the spin of a magnetic impurity located at $\mathbf{r}, s_{i}(\mathbf{r})=\psi^{\dagger}(\mathbf{r}) \sigma^{i} \psi(\mathbf{r})$ is the spin of the surface electrons and $J_{z}, J_{\|}$are the coupling parameters. The Hamiltonians (11) and (2) together describe the problem of magnetic impurities on the surface of a TI, which is the starting point of this paper. This phenomenological Hamiltonian can be derived rigorously from more realistic models. For example, strained HgTe, which is expected to be a TI, can be described by a realistic Kane model [8, 14]. A straightforward derivation, following the similar steps outlined in Ref. [8], gives $\hbar v_{f} \approx 3.4 \mathrm{eV} \cdot \AA, J_{z} \approx-20 \mathrm{meV}$ and $J_{\|} \approx-40 \mathrm{meV}$.

In graphene, the chemical potential is automatically tuned to the Dirac point for a half-filled system. In a TI the chemical potential in general needs to be tuned to the Dirac point by a metallic gate, which screens the Coulomb interaction between the surface electrons to an irrelevant local four fermion interaction. In our current work the Coulomb interaction will be ignored throughout, and our results are applicable to length scales larger than the screening length.

Single magnetic impurity.- Let us start by studying the effect of a single magnetic impurity on the surface states. For simplicity, we assume that the impurity is

SIMES, SLAC National Accelerator Center, 2575 Sand Hill Road, Menlo Park, CA 94309

Work supported in part by US Department of Energy contract DE-AC02-76SF00515. 
located at the origin and treat it as a classical spin. Under such a mean-field approximation, the exchange Hamiltonian is written as $\hat{H}_{e x} \simeq \sum_{\mathbf{r}} M_{i}(\mathbf{r}) s_{i}(\mathbf{r})$ with $M_{i}(\mathbf{r})=J_{i}\left\langle S_{i}\right\rangle \delta(\mathbf{r})$. It should be noticed that the $\delta$ function form of the exchange interaction is only correct in the long-wavelength limit. For the description of local physics the $\delta$-function potential should be regularized as a finite range potential. In the following, we take a square well regularization for the exchange interaction and study the case with the impurity spin polarized in the $z$ direction. In this case the exchange Hamiltonian is reduced to

$$
\hat{H}_{e x}^{\prime}=\sum_{\mathbf{r}} M(\mathbf{r}) s_{z}(\mathbf{r})
$$

where $M(\mathbf{r})=M_{0} \Theta\left(r_{0}-r\right)$ with $\Theta\left(r_{0}-r\right)$ the step function and $M_{0}=J_{z}\left\langle S_{z}\right\rangle$. $r_{0}$ determines the range of the exchange interaction. This problem has azimuthal symmetry and can be solved analytically with Bessel functions [15]. From the analytic solution we find that the wavefunction for $r<r_{0}$ decays for energies $|E|<\left|M_{0}\right|$, and oscillates for energies $|E|>\left|M_{0}\right|$. To study observable consequences of this impurity effect, we calculate the local density of states (LDOS) defined by $\rho_{0}(\mathbf{r}, E)=-\frac{1}{2 \pi} \Im\left\{\operatorname{Tr}\left[G^{R}(\mathbf{r}, \mathbf{r}, E)\right]\right\}$, where $G^{R}\left(\mathbf{r}, \mathbf{r}^{\prime}, E\right)$ is the retarded single-particle Green's function. As shown in Fig. 1 (a) and (b), the LDOS is suppressed in the energy range $|E|<\left|M_{0}\right|$ and spatial range $r<r_{0}$. For $r>r_{0}$ the LDOS converges quickly to the impurity-free value $\frac{|E|}{2 \pi \hbar^{2} v_{f}^{2}}$. Such a LDOS gap induced by a magnetic impurity can be observed by STM experiments, and define a sharp criterion to distinguish the TI surface from other two-dimensional systems with an even number of Dirac cones, such as graphene. In graphene, the two components of the Dirac Hamiltonian represent the pseudospin degree of freedom of the two sub-lattices, which don't couple to magnetic impurities directly. Therefore, no suppression of LDOS will be observed [16].

Another interesting physical quantity is the spin LDOS, defined by $\rho_{i}(\mathbf{r}, E)=-\frac{1}{2 \pi} \Im\left\{\operatorname{Tr}\left[G^{R}(\mathbf{r}, \mathbf{r}, E) \sigma_{i}\right]\right\}$, $i=x, y, z$. Experimentally, the spin LDOS can be measured by the recently developed spin-resolved STM technique 17]. In order to calculate the spin LDOS induced by a magnetic impurity with arbitrary polarization, we can not use the analytic solution discussed above since rotation symmetry is broken. Therefore, we use the T-matrix formalism 18 to calculate the Green's function $G^{R}\left(\mathbf{r}, \mathbf{r}^{\prime}, E\right)$. The calculated distribution of the spin LDOS $\rho_{i}(\mathbf{r}, E)$ at a given energy is shown in Fig. 1 (c) for the out-of-plane and (d) in-plane magnetization of the impurity. As seen from Fig. 1 (c), the $z$-direction magnetization induces not only a $z$-direction spin LDOS, but also an in-plane spin LDOS. This is a direct consequence of the spin-orbit coupling of the surface states. In fact, the Dirac Hamiltonian (1) can be regarded as an electron spin coupled to a momentum-dependent effective magnetic field $\mathbf{B}_{e f f}=\hbar v_{f} \mathbf{k}$. As an electron propagates, its spin processes around $\mathbf{B}_{\text {eff }}$. Since $\mathbf{B}_{e f f}$ is parallel to
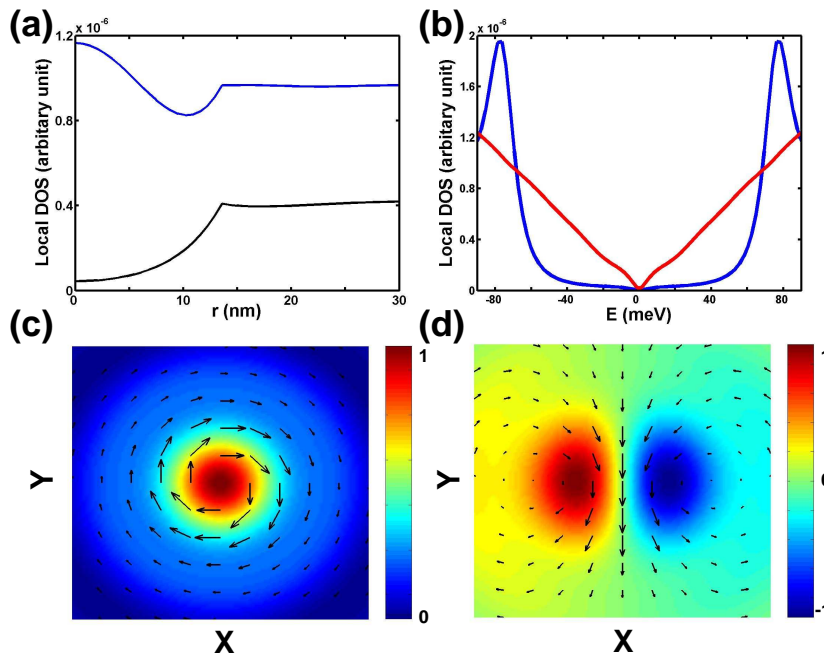

(d)

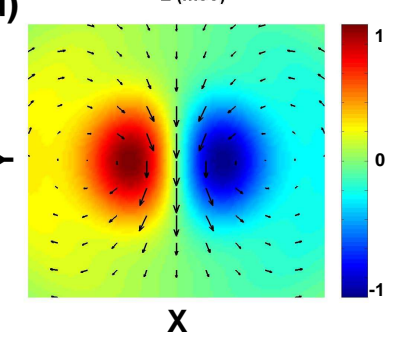

FIG. 1: (a) Charge local density of states (LDOS) as a function of the distance $r$ from the magnetic impurity for electron energies $E=30 \mathrm{meV}$ (black line) and $E=70 \mathrm{meV}$ (blue line). (b) Charge LDOS as a function of electron energy $E$ at positions $r=0$ (blue line) and $r=20 \mathrm{~nm}$ (red line). Here we assume a magnetic impurity strength $M_{0}=50 \mathrm{meV}$ and a coupling range $r_{0}=13 \mathrm{~nm}$. The spin LDOS is plotted as a function of position at $E=10 \mathrm{meV}$ for magnetization of the magnetic impurity placed at the origin $(0,0)$ in (c) the $z$-direction and (d) the $y$-direction. Here the arrow indicates the in-plane spin LDOS and the color shows the $z$-direction spin LDOS.

the propagation direction of the electron, we immediately realize that the spin of the electron will always precess in a plane perpendicular to the direction of propagation. For example, when an electron with spin polarized in the $z$-direction is moving towards the $x$ direction, the spin is precessing in the $y z$ plane. Consequently, the spin LDOS vector $\rho_{s}(\mathbf{r}, E)=\left(\rho_{x}, \rho_{y}, \rho_{z}\right)(\mathbf{r}, E)$ is canted towards the $y$-direction when $\mathbf{r}$ is along the positive $x$-axis. A similar analysis can apply to other directions, from which we can understand the spin LDOS distribution in Fig. 1(c). For an in-plane impurity spin polarized in the $y$-direction, the precession picture remains valid. For example, no precession occurs along the $y$-axis since the spin is precessing in the $x z$ plane, in agreement with Fig. 1(d). The spin LDOS pattern in Fig. 1 (c) and (d) can also be understood by symmetry. When the impurity spin is polarized in the $z$-direction, the system is invariant under the rotations about $z$-axis, and so is the spin LDOS. For an in-plane impurity spin, rotation symmetry is broken, but there is a discrete symmetry defined by a $\pi$-rotation along the $z$-axis combined with a time-reversal transformation. Such a residual symmetry is preserved in the pattern of Fig. 11(d).

Besides the patterns discussed above which are due to spin precession, another important feature of the spin LDOS is the longitudinal decay $\rho_{i} \propto 1 / r^{2}$. As a result 
of integrating over energies below the Fermi energy, the local spin polarization $\langle\mathbf{s}(\mathbf{r})\rangle$ will behave as $1 / r^{3}$. Such a $1 / r^{3}$ power law is a direct consequence of the fact that the spatial scaling dimension of the spin density in the free Dirac fermion theory is 2 .

Random magnetic impurities.- In the following we will focus on the behavior of the system with randomly distributed magnetic impurities on the surface $\mathbf{S}(\mathbf{r})=$ $\sum_{i} \mathbf{S}_{i} \delta\left(\mathbf{r}-\mathbf{R}_{i}\right)$, where $\mathbf{R}_{i}$ are the positions of magnetic impurities. As every magnetic impurity will open a local gap in its vicinity, we may expect the system to be gapped everywhere, at least at the mean field level. However, this is not necessarily true if the magnetization of the magnetic impurities is nonuniform. To see this, we consider again the mean-field form of the exchange Hamiltonian (3) with a magnetization domain wall along the $y$-axis at $x=0$, which is given by $M(x)>0(M(x)<0)$ for $x>0(x<0)$. Solving the Schrödinger equation directly on the domain wall, we obtain gapless chiral fermion modes along the domain wall with wave function $\psi \sim(1, i)^{T} \exp \left[i k_{y} y-\int_{0}^{x} \frac{M(x)}{\hbar v_{f}} d x\right]$ and energy dispersion $E=\hbar v_{f} k_{y}$. Thus, this system is in fact not totally gapped but has gapless modes. Compared with the fully gapped system, the appearance of such gapless modes will cost more energy. Therefore, heuristically we expect the system not to favor any magnetic domain wall, which indicates that magnetic impurities should be ferromagnetically coupled.

Keeping such a heuristic picture in mind, we now study the coupling between two magnetic impurities microscopically. The itinerant electrons can mediate a spin interaction between two magnetic impurities, known as the Ruderman-Kittel-Kasuya-Yosida (RKKY) interaction. Such a coupling can be obtained by integrating out the fermions in the Hamiltonians (11) and (2), which results in the form $\hat{H}_{i n}=\sum_{i, j=x, y, z} \Phi_{i, j}\left(\left|\mathbf{r}-\mathbf{r}^{\prime}\right|\right) S_{1 i}(\mathbf{r}) S_{2 j}\left(\mathbf{r}^{\prime}\right)$ for any two magnetic impurities $S_{1}$ and $S_{2}$. The coupling constant $\Phi_{i j}(R)$ is a function of $R=\left|\mathbf{r}-\mathbf{r}^{\prime}\right|$ and can be extracted from standard second-order perturbation theory. For example, the $z$-direction coupling constant has the form $\Phi_{z z}(R)=\frac{J_{z}^{2} a_{0}^{4}}{\hbar v_{f} R^{3}}\left(F_{+}\left(k_{F} R\right)+F_{-}\left(k_{F} R\right)\right)$, where $k_{F}$ is the Fermi momentum, $a_{0}$ is the lattice constant and $F_{+(-)}\left(x_{F}\right)=\int_{0}^{x_{F}\left(x_{c}\right)} \frac{x d x}{2 \pi} \int_{x_{F}}^{x_{c}} \frac{x^{\prime} d x^{\prime}}{2 \pi} \frac{1}{+(-) x-x^{\prime}}\left[J_{0}(x) J_{0}\left(x^{\prime}\right)\right.$ $\left.-(+) J_{1}(x) J_{1}\left(x^{\prime}\right)\right] . \quad J_{n}(x)$ is the Bessel function and $x_{c}=k_{c} R$ with $k_{c}$ a large momentum cutoff. The oscillating part of the RKKY interaction is determined by $F_{+}+F_{-}$and the decaying part is proportional to $1 / R^{3}$. Such dependence is related to the $1 / r^{3}$ dependence of the local spin polarization induced by a single magnetic impurity discussed earlier. Being a consequence of the Dirac Hamiltonian, similar behavior has also been found in graphene[19, 20]. Moreover, $F_{+}$and $F_{-}$give the intra-band and inter-band contributions respectively. The novel property of Dirac fermions appears when the chemical potential is close to the Dirac point. The oscillation period of the RKKY interaction being determined by the Fermi wavelength $\lambda_{F}=1 / k_{F}$, the os-

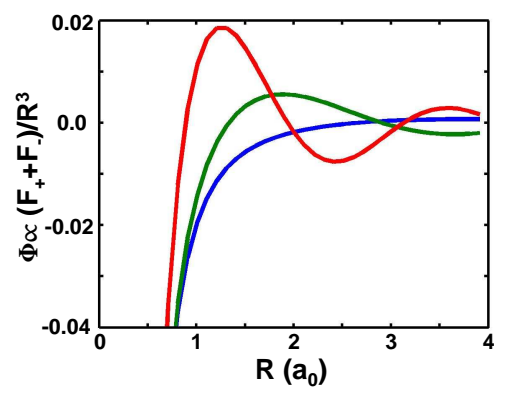

FIG. 2: RKKY interaction versus the distance $R$ between two magnetic impurities. Fermi momentum $k_{F}$ is chosen to be $0.5 / a_{0}$ for the blue line, $1.0 / a_{0}$ for the green line and $1.5 / a_{0}$ for the red line, where $a_{0}$ is the lattice constant.

cillation becomes weaker as $k_{F} \rightarrow 0$, as shown in Fig. 2. Eventually the two magnetic impurities become ferromagnetically coupled when $\lambda_{F}$ is much larger than the average distance between them. The RKKY interaction mediated by the surface states of a TI is quite different from that between magnetic impurities in graphene. The tendency towards ferromagnetic 21, 22] or antiferromagnetic correlations [19, 20, 23] in graphene is a topic of current debate. Considering only the noninteracting lattice model for graphene [19, 20], it is found that the RKKY interaction is ferromagnetic for local moments within equivalent sublattices but antiferromagnetic for opposite sublattices. Such different behavior is again a consequence of the different physical meaning of the two components of the Dirac spinor in the two systems.

Due to the ferromagnetic RKKY interaction discussed above, we expect ferromagnetic order to appear in this system when the chemical potential is near the Dirac point. At the mean field level, from the $\hat{H}_{e x}^{z}$ term in (2) we know that $J_{z}\left\langle s_{z}\right\rangle$ acts as an effective magnetic field to magnetize the magnetic impurities. At the same time, $J_{z}\left\langle S_{z}\right\rangle$ acts as the effective magnetic field to polarize the electron spin. The behavior of the critical temperature $T_{c}$ for the ferromagnetism can be extracted from a standard calculation [24], and is given by $k_{B} T_{c}=\frac{S_{0}\left(S_{0}+1\right) a_{0}^{2} y}{6 \pi \hbar^{2} v_{f}^{2}} J_{z}^{2}\left(E_{c}-E_{f}\right)$, where $E_{c}$ is a cutoff energy, $E_{f}$ is the Fermi energy, and $S_{0}$ is the saturation spin value of each magnetic impurity. Setting $y=0.1, a_{0}=6 \AA, E_{c}=0.1 \mathrm{eV}$ and $S_{0}=5 / 2$ for $\mathrm{Mn}^{2+}, T_{c}$ is estimated to be of the order of $\sim 0.1$ K. Interestingly, such a ferromagnetically ordered surface state also carries a half quantized Hall conductance $\sigma_{H}= \pm e^{2} / 2 h$, which can be understood as the parity anomaly of massless Dirac fermions [25, 26], and is a direct manifestation of the nontrivial topology of the bulk system. To go beyond the mean-field picture studied above, we now switch gears to a renormalization group (RG) approach with more generic quenched disorder. We consider three types of random potentials: random charge potential coupled to $\psi^{\dagger} \psi$, random XY-moment 
coupled to $\psi^{\dagger} \sigma^{x(y)} \psi$, and random Z-moment coupled to $\psi^{\dagger} \sigma^{z} \psi$. The Lagrangian density including all the random potentials is $\mathcal{L}=\psi^{\dagger}\left(\partial_{\tau}+i v_{f} \sigma^{j} \partial_{j}\right) \psi+g_{1} B_{1}(\mathbf{r}) \psi^{\dagger}(\mathbf{r}) \psi(\mathbf{r})-$ $v_{f} g_{2} B_{2}(\mathbf{r})_{j} \psi^{\dagger}(\mathbf{r}) \sigma^{j} \psi(\mathbf{r})+v_{f}^{2} g_{3} B_{3}(\mathbf{r}) \psi^{\dagger}(\mathbf{r}) \sigma^{z} \psi(\mathbf{r})$, where $j=x, y . B_{a}(\mathbf{r})$ represents three types of random potentials with correlation $\left\langle B(\mathbf{r}) B\left(\mathbf{r}^{\prime}\right)\right\rangle=\delta^{2}\left(\mathbf{r}-\mathbf{r}^{\prime}\right) /(2 \pi) . g_{a}^{2}$ is proportional to the standard deviation of each type of random potential distribution, which from naive powercounting is a marginal perturbation. Since the random potential has long range temporal correlation but short range spatial correlation, Lorentz invariance of the bare Dirac Lagrangian is lost, and the Fermi velocity $v_{f}$ flows under RG. In the calculation we assume that the standard deviation $\Delta_{a} \sim g_{a}^{2}$ is small. After integrating out degrees of freedom between momentum cutoff $\tilde{\Lambda}$ and $\Lambda$, the leading order RG equations read

$$
\begin{aligned}
\frac{d v_{f}}{d \ln l} & =-\frac{g_{1}^{2}}{4 \pi^{2} v_{f}}-\frac{g_{2}^{2} v_{f}}{2 \pi^{2}}-\frac{g_{3}^{2} v_{f}^{3}}{4 \pi^{2}} \\
\frac{d Z_{\psi}}{d \ln l} & =-\frac{g_{1}^{2}}{4 \pi^{2} v_{f}^{2}}-\frac{g_{2}^{2}}{2 \pi^{2}}-\frac{g_{3}^{2} v_{f}^{2}}{4 \pi^{2}} \\
\frac{d Z_{v 0}}{d \ln l} & =\frac{g_{1}^{2}}{4 \pi^{2} v_{f}^{2}}+\frac{g_{2}^{2}}{2 \pi^{2}}+\frac{g_{3}^{2} v_{f}^{2}}{4 \pi^{2}} \\
\frac{d Z_{v z}}{d \ln l} & =-\frac{g_{1}^{2}}{4 \pi^{2} v_{f}^{2}}+\frac{g_{2}^{2}}{2 \pi^{2}}-\frac{g_{3}^{2} v_{f}^{2}}{4 \pi^{2}} \\
\frac{d Z_{v x y}}{d \ln l} & =0
\end{aligned}
$$

$Z_{\psi}$ is the wavefunction renormalization and $Z_{v 0}, Z_{v x y}$ and $Z_{v z}$ are vertex corrections to $\psi^{\dagger} \psi, \psi^{\dagger} \sigma^{x(y)} \psi$ and $\psi^{\dagger} \sigma^{z} \psi$ respectively. The complete solution of the RG equations (4) is complicated, but the solutions in different limits can be obtained straightforwardly. For instance, if $g_{1}=g_{3}=0$, the solution of the RG equations reads:

$$
\begin{gathered}
z=1+\frac{g_{2}^{2}}{2 \pi^{2}}, \Delta\left[v_{f}\right]=\frac{g_{2}^{2}}{2 \pi^{2}}, \Delta\left[\psi^{\dagger} \psi\right]=2, \\
\Delta\left[\psi^{\dagger} \sigma^{z} \psi\right]=2, \Delta\left[\psi^{\dagger} \sigma^{x(y)} \psi\right]=2+\frac{g_{2}^{2}}{2 \pi^{2}} .
\end{gathered}
$$

The coupling constant $g_{2}$ does not flow under RG because all the corrections can be absorbed into $v_{f}$. The dynamical scaling dimension $z$ is changed because the Fermi velocity acquires a nonzero scaling dimension under RG, leading to the following scaling of the LDOS: $\rho(\omega) \sim \omega^{\frac{2-z}{z}}$ [27]. Using the scaling dimensions of the fermion bilinears listed in Eq. 5, the long distance spin distribution around an isolated magnetic impurity with in-plane moment is given by $S^{x(y)} \delta^{2}(r): s^{x(y)}(r)=$ $\psi^{\dagger} \sigma^{x(y)} \psi(r) \sim \frac{1}{r^{3+g_{2}^{2} / \pi^{2}}}$. Thus the spin LDOS pattern remains similar to that shown in Fig. 1 (c) and (d), but decays with a different power law.

Quenched disorder has been studied in graphene, with $N=4$ flavors of Dirac fermions [28, 29, 30]. In this case, ripples of a graphene sheet, interpreted as a random "gauge potential" $B_{2}(x)_{j} \psi^{\dagger} \sigma^{j} \psi$, have attracted most of the attention. In contrast with our results, the $1 / N$ expansion is usually taken when the RG equations are derived for graphene. The competition between random potentials and the Coulomb interaction have also been studied in graphene. In this case, the RG equations lead to various nontrivial fixed points [31].

In conclusion, we have investigated the effects of magnetic impurities on the surface states of a TI. A magnetic impurity breaks time reversal symmetry and suppresses the low energy LDOS locally. The surface states mediate a coupling between the magnetic impurities which is always ferromagnetic when the chemical potential lies close to the Dirac point. Therefore, we expect that a finite concentration of magnetic impurities would give rise to a ferromagnetic ground state on the surface. This mechanism provides a physical realization of the novel topological magneto-electric effect discussed in Ref. [8] , which requires breaking of time reversal symmetry on the surface of a TI. We also investigated the effect of quenched impurities on the surface states and presented the RG equations governing the flow of the coupling constants. The distinct signatures of magnetic impurities on the surface states of a TI discussed in this work can be readily observed in STM experiments, possibly on the surface of $\mathrm{Bi}_{\mathrm{x}} \mathrm{Sb}_{1-\mathrm{x}}$ [32] or strained $\mathrm{HgTe}$.

The authors would like to thank T.L. Hughes, J. Maciejko, T. X. Ma, S. Raghu, S. Ryu and B. F. Zhu for helpful discussions. This work is supported by the NSF under grant numbers DMR-0342832 and the US Department of Energy, Office of Basic Energy Sciences under contract DE-AC03-76SF00515. We also acknowledge financial support from the Focus Center Research Program (FCRP) Center on Functional Engineered Nanoarchitectonics (FENA). CXL acknowledges CSC, NSF (Grant No.10774086, 10574076) and Basic Research Development of China (Grant No. 2006CB921500).
[1] C. L. Kane and E. J. Mele, Phys. Rev. Lett. 95, 226801 (2005).

[2] B.A. Bernevig and S.C. Zhang, Phys. Rev. Lett. 96, 106802 (2006).

[3] B. A. Bernevig, T. L. Hughes, and S.-C. Zhang, Science 314, 1757 (2006).

[4] M. Konig, S. Wiedmann, C. Brune, A. Roth, H. Buh- mann, L. W. Molenkamp, X.-L. Qi, and S.-C. Zhang, Science 318, 766 (2007).

[5] L. Fu, C. L. Kane, and E. J. Mele, Phys. Rev. Lett. 98, 106803 (2007).

[6] J. E. Moore and L. Balents, Phys. Rev. B 75, 121306 (2007)

[7] R. Roy, cond-mat/0607531 (2006). 
[8] X.-L. Qi, T. Hughes, and S.-C. Zhang, Arxiv: condmat/0802.3537 (2008).

[9] C. Wu, B. A. Bernevig, and S.-C. Zhang, Phys. Rev. Lett. 96, 106401 (2006).

[10] C. Xu and J. Moore, Phys. Rev. B 73, 045322 (2006).

[11] G. W. Semenoff, Phys. Rev. Lett. 53, 2449 (1984).

[12] K. S. Novoselov, A. K. Geim, S. V. Morozov, D. Jiang, M. I. Katsnelson, I. V. Grigorieva, and S. V. Dubonos, Nature 438, 197 (2005).

[13] X.-L. Qi, T. L. Hughes, and S.-C. Zhang, Nat Phys 4, 273 (2008).

[14] X. Dai, T. L. Hughes, X.-L. Qi, Z. Fang, and S.-C. Zhang, Phys. Rev. B 77, 125319 (2008).

[15] A. Matulis and F. M. Peeters, Phys. Rev. B 77, 115423 (2008).

[16] T. O. Wehling, A. V. Balatsky, M. I. Katsnelson, A. I. Lichtenstein, K. Scharnberg, and R. Wiesendanger, Phys. Rev. B 75, 125425 (2007).

[17] F. Meier, L. Zhou, J. Wiebe, and R. Wiesendanger, Science 320, 82 (2008).

[18] H. Shiba, Prog. Theor. Phys. 40, 435 (1968).

[19] L. Brey, H. A. Fertig, and S. D. Sarma, Phys. Rev. Lett. 99, 116802 (2007).

[20] S. Saremi, Phys. Rev. B 76, 184430 (2007).
[21] M. A. H. Vozmediano, M. P. Lopez-Sancho, T. Stauber, and F. Guinea, Phys. Rev. B 72, 155121 (2005).

[22] V. Dugaev, V. I. Litvinov, and J. Barnas, PRB 74, 224438 (2006).

[23] L. Pisani, J. A. Chan, B. Montanari, and N. M. Harrison, Phys. Rev. B 75, 064418 (2007).

[24] T. Jungwirth, J. Sinova, J. Masek, J. Kucera, and A. H. MacDonald, Rev. Mod. Phys. 78, 809 (2006).

[25] E. Fradkin, E. Dagotto, and D. Boyanovsky, Phys. Rev. Lett. 57, 2967 (1986).

[26] K. Nomura, S. Ryu, M. Koshino, C. Mudry, and A. Furusaki, Physical Review Letters 100, 246806 (2008).

[27] A. W. Ludwig, M. P. Fisher, R. Shankar, and G. Grinstein, Phys. Rev. B 50, 7526 (1994).

[28] T. Stauber, F. Guinea, and M. A. H. Vozmediano, Phys. Rev. B 71, 041406 (2005).

[29] O. Vafek and M. J. Case, Phys. Rev. B 77, 033410 (2008).

[30] I. F. Herbut, V. Juricic, and O. Vafek, Phys. Rev. Lett. 100, 046403 (2008).

[31] M. S. Foster and I. L. Aleiner, Phys. Rev. B 77, 195413 (2008).

[32] D. Hsieh, D. Qian, L. Wray, Y. Xia, Y. S. Hor, R. J. Cava, and M. Z. Hasan, Nature 452, 970 (2008). 